\title{
Detecting entanglement of two-electron spin qubits with witness operators
}

\author{
A. Borras and M. Blaauboer \\ Delft University of Technology, Kavli Institute of Nanoscience, Department of Quantum \\ Nanoscience, Lorentzweg 1, NL-2628 CJ Delft, The Netherlands
}

(Received 12 May 2011; published 6 July 2011)

\begin{abstract}
We propose a scheme for detecting entanglement between two-electron spin qubits in a double quantum dot using an entanglement witness operator. We first calculate the optimal configuration of the two electron spins, defined as the position in the energy level spectrum where, averaged over the nuclear spin distribution, (1) the probability to have two separated electrons and (2) the degree of entanglement of the quantum state quantified by the concurrence are both large. Using a density matrix approach, we then calculate the evolution of the expectation value of the witness operator for the two-spin singlet state, taking into account the effect of decoherence due to quantum charge fluctuations modeled as a boson bath. We find that, for large interdot coupling, it is possible to obtain a highly entangled and robust ground state.
\end{abstract}

DOI: 10.1103/PhysRevB.84.033301

PACS number(s): 73.21.La, 03.67.Bg, 03.67.Mn

Introduction. Entanglement-nonclassical correlations between quantum-mechanical particles-has for a long time been a theoretically predicted concept ${ }^{1}$ without experimental proof. The first experimental observation of entanglement was reported for photon pairs in $1972,{ }^{2}$ and since then entanglement between protons, ${ }^{3}$ kaons, ${ }^{4}$ trapped ions, ${ }^{5}$ and individual neutrons, ${ }^{6}$ between an atom and a photon, ${ }^{7}$ and between superconducting qubits ${ }^{8}$ has also been demonstrated. An important goal in present-day solid-state quantum physics is to generate and detect (prove) entanglement between individual electrons. The motivation behind this quest comes both from the fact that entanglement between electrons in a solid-state structure has not yet been demonstrated and from the recent experimental progress in the field of quantum information processing in these systems, ${ }^{9}$ which has, among other things, led to experimental realization of single- and two-qubit manipulations of electron spin qubits in quantum $\operatorname{dots}^{10,11}$ and coherent control of spins in diamond. ${ }^{12}$

In this work we propose a scheme for detecting entanglement in the former system, namely, entanglement of two-electron spin qubits in a double quantum dot (DQD). Many aspects of this quantum system, such as hyperfine coupling to the nuclear spins, ${ }^{13-15}$ the spin blockade, ${ }^{16,17}$ and the effects of applying a slanting magnetic field ${ }^{18}$ are currently active topics of research. Our proposal consists of preparing and tuning the two-spin system such that the ground state contains a large entangled component and measuring a so-called entanglement witness operator ${ }^{19}$ to demonstrate the presence of this entanglement. Entanglement witnesses are Hermitian operators that are designed to detect a specific entangled state. Their expectation value is positive for all separable quantum states (in the class of states considered) and negative for at least one entangled state, usually the state the experiment aims to create. Entanglement witnesses have been used to detect entangled states in trapped ion systems or entangled photon states. ${ }^{20}$

Theoretical proposals for detecting entanglement between individual electrons using witness operators are scarce. The authors of Ref. 21 present a proposal for implementing witness operators to detect electron-hole entanglement in multiterminal conductors in the presence of noise due to random accumulated phases. In a previous work, ${ }^{22}$ we have proposed a turnstile mechanism as a suitable setup for demonstrating entanglement between two electron spins in a DQD, assuming phenomenological decoherence times for the spin relaxation ( $T_{1}$ time) and decoherence $\left(T_{2}\right.$ time). A short-lived entangled singlet state can be generated in this system by fast adiabatic passage. ${ }^{11}$ However, because of hyperfine interaction this singlet state is rapidly mixed with the triplet state with typical dephasing time of $10 \mathrm{~ns}$. Here, we propose a different approach in which slow adiabatic passage is used to drive the system to a configuration where the ground state is highly entangled. This technique allows us to obtain a state which remains entangled for longer times. We explicitly consider the influence of the hyperfine interaction and the decoherence due to quantum charge fluctuations, which are caused by changing gate voltages to control the system. Starting from the two-electron Hamiltonian, and assuming a large external magnetic field, we first calculate the energy levels and eigenstates in the three-dimensional Hilbert space spanned by the $|S(0,2)\rangle,|S(1,1)\rangle$, and $\left|T_{0}(1,1)\right\rangle$ eigenstates, where $|S(n, m)\rangle\left(\left|T_{0}(n, m)\right\rangle\right)$ represents the singlet (triplet) state with $n$ electrons in the left and $m$ electrons in the right dot. We then average over the nuclear field components and calculate the average probability of having one electron in each dot, $\left\langle P_{11}\right\rangle_{\text {nucl }}$, and the concurrence $\langle C\rangle_{\text {nucl }}$ of the entangled component of the ground state. This allows us to determine the "optimal" configuration in the level diagram in which both of the latter quantities are large. We then investigate the effects of decoherence of the entangled quantum state due to quantum charge fluctuations, modeled as a boson bath. Using the Born-Markov approximation, we calculate the reduced density matrix $\rho(t)$ of the system and the evolution of the expectation value of the witness operator, $\operatorname{Tr}[W \rho(t)]$. We find the optimal values in our parameter space that provide a highly entangled ground state which is also robust under the action of charge fluctuations.

Model. We consider a charge configuration of the DQD where each electron can be located in a different quantum dot $(1,1)$, or both of them in the right dot $(0,2)$. In the $(1,1)$ configuration the four accessible states are the singlet $|S(1,1)\rangle=$ $(|\uparrow \downarrow\rangle-|\downarrow \uparrow\rangle) / \sqrt{2}$ and the three triplet states $\left|T_{0}(1,1)\right\rangle=$ $(|\uparrow \downarrow\rangle+|\downarrow \uparrow\rangle) / \sqrt{2},\left|T_{+}\right\rangle=|\uparrow \uparrow\rangle$ and $\left|T_{-}\right\rangle=|\downarrow \downarrow\rangle$. In the 
$(0,2)$ configuration only the singlet $|S(0,2)\rangle$ can be populated, the triplet states $\left|T_{i}(0,2)\right\rangle$ having much higher energies. The spin-preserving part of the Hamiltonian is given by $H_{0}=$ $\Delta\left|S_{02}\right\rangle\left\langle S_{02}\right|+t\left(\left|S_{02}\right\rangle\left\langle S_{11}|+| S_{11}\right\rangle\left\langle S_{02}\right|\right)$.

The tunneling parameter $t$ couples the two singlets, allowing one electron to transfer between the two dots, while $\Delta$ is the energy difference between the $(0,2)$ and the $(1,1)$ singlets. Both parameters can be externally controlled by changing gate voltages and are used to tune the system to the desired configuration. In addition, the hyperfine interaction of the electrons with the nuclear spins mixes the singlet and triplet states in the $(1,1)$ configuration. Each electron interacts with a large number of nuclear spins in the left $(L)$ and right $(R)$ dots. The global action of these nuclear spins can be included in a single operator $\hat{\mathbf{B}}_{L, R}^{\text {nucl }}$ which allows us to treat the hyperfine interaction as the interaction between the electrons and this apparent magnetic field. We assume that this nuclear magnetic field remains unchanged over the typical time scale of the electron spin evolution ${ }^{23}$ and hence it can be treated as a classical magnetic field. The combined action of the nuclear magnetic field and of an external magnetic field $B_{\text {ext }}$ is given by

$$
\begin{aligned}
H_{\text {spin }}= & B_{s}^{z}\left(\left|T_{+}\right\rangle\left\langle T_{+}|-| T_{-}\right\rangle\left\langle T_{-}\right|\right) \\
& +\left(B_{a}^{z}\left|T_{0}\right\rangle\left\langle S_{11}\left|+\frac{B_{s}^{x} \pm i B_{s}^{y}}{\sqrt{2}}\right| T_{0}\right\rangle\right. \\
& \left.\times\left\langle T_{ \pm}\left|+\frac{\mp B_{a}^{x}-i B_{a}^{y}}{\sqrt{2}}\right| S_{11}\right\rangle\left\langle T_{ \pm}\right|+\text {H.c. }\right),
\end{aligned}
$$

where $\mathbf{B}_{a} \equiv\left(\mathbf{B}_{L}^{\text {nucl }}-\mathbf{B}_{R}^{\text {nucl }}\right) / 2$ and $\mathbf{B}_{s} \equiv\left(\mathbf{B}_{L}^{\text {nucl }}+\mathbf{B}_{R}^{\text {nucl }}\right) / 2+$ $B_{\text {ext }} \mathbf{z}$. The total Hamiltonian of the system is then given by $\hat{H}_{D Q D}=\hat{H}_{0}+\hat{H}_{\text {spin }}{ }^{17}$ and its dynamics can be externally controlled by tuning $\Delta, t$, and $B_{\text {ext }}$. Assuming a large external magnetic field $B_{\text {ext }}$, the triplet states $\left|T_{i}\right\rangle$ are split off by the Zeeman energy, and the state space is reduced to $\left\{\left|T_{0}\right\rangle,|S(1,1)\rangle,|S(0,2)\rangle\right\}$. Under these circumstances it is possible to derive an effective Hamiltonian ${ }^{15}$

$$
H_{\text {eff }}=\Delta\left|S_{02}\right\rangle\left\langle S_{02}|+t| S_{02}\right\rangle\left\langle S_{11}|+M| S_{11}\right\rangle\left\langle T_{0}\right|+\text { H.c., }
$$

where $M \equiv B_{a}^{z}+\left[\left(B_{s}^{x}+i B_{s}^{y}\right)\left(B_{a}^{x}-i B_{a}^{y}\right)+\right.$ c.c. $] / 2 B_{s}^{z}$. The parameter $M$ includes the effects of the hyperfine interaction. The eigenstates of the Hamiltonian (2) are given by

$$
\left|\psi_{i}\right\rangle=\frac{\frac{M}{E_{i}}\left|T_{0}\right\rangle+\left|S_{11}\right\rangle+\frac{t}{E_{i}-\Delta}\left|S_{02}\right\rangle}{\sqrt{1+\frac{M^{2}}{E_{i}^{2}}+\frac{t^{2}}{\left(E_{i}-\Delta\right)^{2}}}},
$$

where $E_{i}$ are their corresponding energies $\left(E_{0}<E_{1}<\right.$ $\left.E_{2}\right)$. These energies are given by $E_{0(1)}=\Delta / 3-\left(s_{1}+\right.$ $\left.s_{2}\right) / 2+(-) i \sqrt{3}\left(s_{1}-s_{2}\right) / 2$ and $E_{2}=\Delta / 3+s_{1}+s_{2}$, with $s_{1,2}=\left(r \pm \sqrt{q^{3}+r^{2}}\right)^{1 / 3}, \quad r=\Delta\left(-18 M^{2}+9 t^{2}+2 \Delta^{2}\right) / 54$, and $q=-\left(3\left(M^{2}+t^{2}\right)+\Delta^{2}\right) / 9 .^{24}$

The entangled component of the ground state is given by its projection onto the $(1,1)$ subspace,

$$
\left|\psi_{0}^{11}\right\rangle=\left(1+M^{2} / E_{0}^{2}\right)^{-1 / 2}\left(\frac{M}{E_{0}}\left|T_{0}\right\rangle+\left|S_{11}\right\rangle\right) .
$$

The amount of entanglement of $\left|\psi_{0}^{11}\right\rangle$ can be quantified by the concurrence. ${ }^{25}$ Both the concurrence $C$ and the probability
$P_{11}$ of having one electron in each dot must be large to have a highly entangled state. Their mathematical expressions are given by

$$
C=\frac{1-M^{2} / E_{0}^{2}}{1+M^{2} / E_{0}^{2}}, \quad P_{11}=1-\frac{\frac{t^{2}}{\left(E_{0}-\Delta\right)^{2}}}{1+\frac{M^{2}}{E_{0}{ }^{2}}+\frac{t^{2}}{\left(E_{0}-\Delta\right)^{2}}} .
$$

The concurrence $C$ is maximal when $\left|E_{0}\right| \gg M$, while $P_{11}$ is maximal in the presence of a large detuning $\left(\Delta \gg\left|E_{0}\right|\right)$. Both conditions can be simultaneously fulfilled only for intermediate values of $\Delta$ where $\Delta \gg\left|E_{0}\right| \gg M$ (typically $M \sim 0.1-1 \mu \mathrm{eV})$. To find the optimal $\Delta$ and $t$ values we consider the maximization of their product $\xi=P_{11} C$.

Averaging over the nuclear field distribution. Given the random character of the nuclear magnetic field, any observable can be calculated by taking the average over the probability distribution that characterizes $\mathbf{B}_{L, R}^{\text {nucl }}$. This distribution is Gaussian with variance $\left\langle\mathbf{B}_{L, R}^{\text {nucl }}\right\rangle=$ $E_{n}^{2} / N_{\text {eff }}$, with $E_{n} \approx 0.135 \mathrm{meV}$ for GaAs and $N_{\text {eff }} \approx$ $10^{6}$ the effective number of nuclei in typical dots. ${ }^{17,26}$ For external magnetic fields $B_{\text {ext }}>2.5 \mu \mathrm{eV}(100 \mathrm{mT})$ the transversal components of the nuclear magnetic field $\left(B_{x, y}^{\text {nucl }}\right)$ are negligible and the hyperfine interaction parameter $M$ is mainly given by the difference of the $z$ components of the nuclear magnetic fields $\left(M \simeq B_{a}^{z}\right.$ ). The probability distribution for $M$ is then approximately Gaussian with variance $\sigma^{2}=$ $\left\langle\mathbf{B}_{L, R}^{\text {nucl }}\right\rangle / 2$.

Figure 1 shows the averaged success rate $\langle\xi\rangle_{\text {nucl }}$ obtained using the previous approximated Gaussian distribution for $M$. The entanglement of the ground state is low for $\Delta<0$ where $P_{11}<0.5$, and for small values of the interdot coupling $t$ where the ground state is nearly a separable state. The black line links the optimal values $\left\{\Delta_{\text {opt }}, t_{\text {opt }}\right\}$ that maximize $\langle\xi\rangle_{\text {nucl }}$. For $t_{\mathrm{opt}}>5 \mu \mathrm{eV}$, and in a large region nearby, the ground state is highly entangled $\left(\langle\xi\rangle_{\text {nucl }}>0.95\right)$ up to values of 0.99 for $t_{\mathrm{opt}}=20 \mu \mathrm{eV}$. Obtaining the average over the nuclear probability distribution is usually a difficult task, but in this case the value of the hyperfine parameter $M$ in Eqs. (5) can to a good approximation be substituted by the standard deviation of its Gaussian probability distribution $\left[\langle\xi(M)\rangle_{\text {nucl }} \approx \xi\left(\sqrt{\left\langle M^{2}\right\rangle}\right)=\xi(\sigma)\right]$. We have checked that this approximation holds in the highly entangled region and fails only for small $t$ and large $\Delta$. In that region $E_{0} \simeq M$, and small variations in $M$ have a great impact on the (small) value of the concurrence $C$, and hence on $\xi$.

The entanglement of the system can be detected using witness operators. For an entangled state $|\varphi\rangle=\gamma_{1}\left|T_{0}\right\rangle+$ $\gamma_{2}|S(1,1)\rangle$ (with $\gamma_{2}>\gamma_{1}$ ) the optimal operator to detect its entanglement is given by $W=\left|T_{0}\right\rangle\left\langle T_{0}|+| S(1,1)\right\rangle\langle S(1,1)|+$ $\left|T_{0}\right\rangle\langle S(1,1)|+| S(1,1)\rangle\left\langle T_{0}\right|{ }^{20}$ Its expectation value is given by $\operatorname{Tr}(W|\varphi\rangle\langle\varphi|)=\left(\gamma_{1}^{2}-\gamma_{2}^{2}\right) / 2$, which is proportional to the concurrence $[\operatorname{Tr}(W|\varphi\rangle\langle\varphi|)=-C / 2]$. This witness can be rewritten as $W=I / 2-|S(1,1)\rangle\langle S(1,1)|$. In order to measure the expectation value of $W$, it is then enough to measure the probability $P[S(1,1)]$ of the ground state to be the $|S(1,1)\rangle$ state. In a DQD this probability can easily be measured using a quantum point contact (QPC). ${ }^{9}$ Our proposal to detect the entanglement is then the following: to initialize the system in the $|S(0,2)\rangle$ state (point $A$ in Fig. 1) and adiabatically increase $\Delta$ to the optimal position $\left\{\Delta_{\text {opt }}, t_{\text {opt }}\right\}$ (point $B$ in Fig. 1). A 


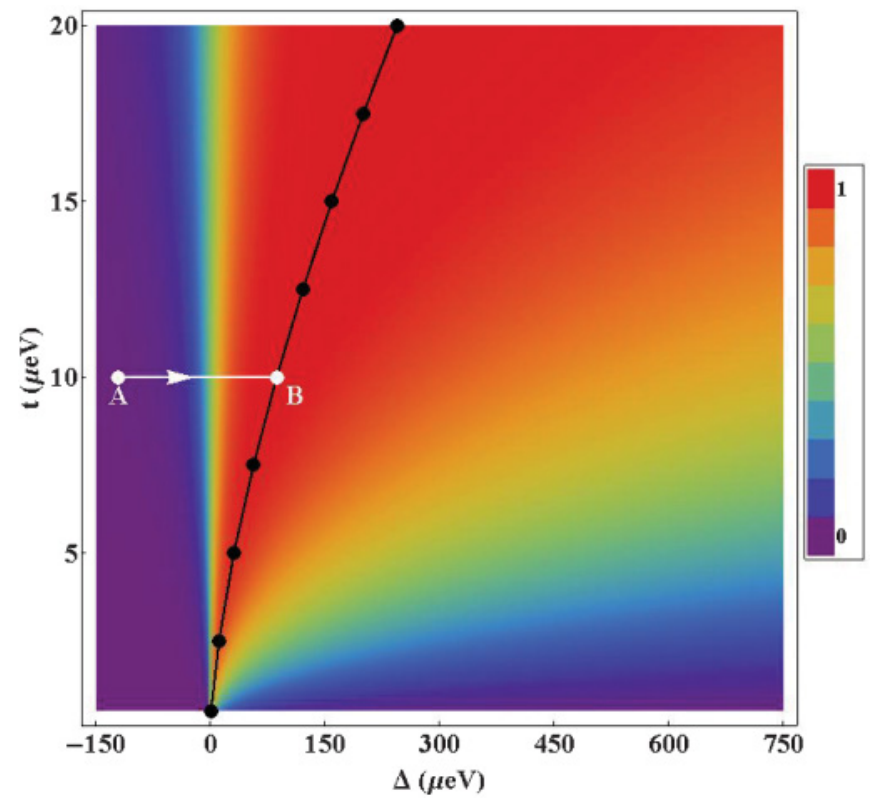

FIG. 1. (Color online) Averaged entanglement probability $\langle\xi\rangle_{\text {nucl }}$. The black line represents the optimal configuration $\left\{\Delta_{\text {opt }}, t_{\text {opt }}\right\}$ that maximizes the entanglement of the system. The white solid line represents the trajectory followed from the initial state $A$ to the final state $B$.

QPC can then be used to measure $\mathrm{P}(\mathrm{S}(1,1))$ and obtain the expectation value of the witness operator.

Entanglement time evolution. When modifying gate voltages to drive the system to its optimal position and to measure the probability $P[S(1,1)]$, the resulting charge fluctuations cause the tunnel coupling $t$ and energy offset $\Delta$ to fluctuate. The inset of Fig. 2 shows that $E_{\text {gap }} \geqslant k_{B} T$ for $T=10 \mathrm{mK}$ and $t_{\mathrm{opt}} \geqslant 5 \mu \mathrm{eV}$, (i.e., in the highly entangled region) so that quantum fluctuations are important. ${ }^{27}$ In the last part, we explore the influence of these fluctuations on the time evolution of the expectation value of the entanglement witness.

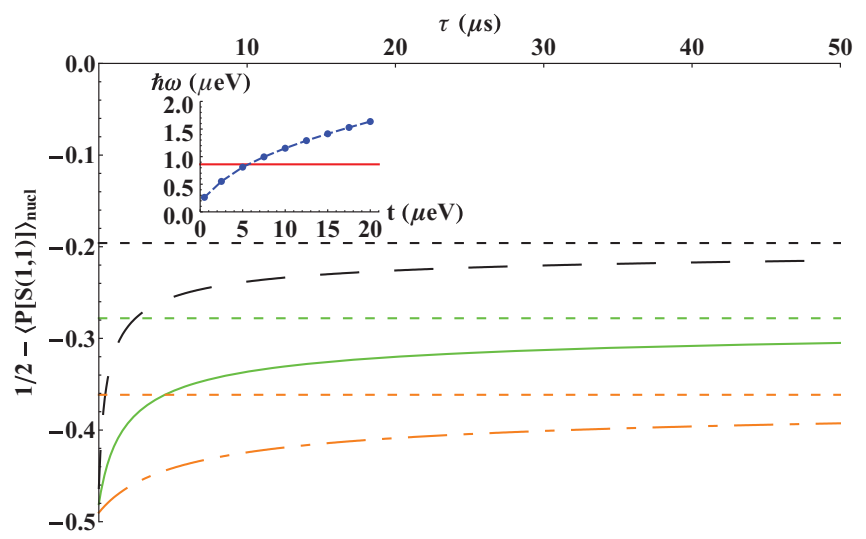

FIG. 2. (Color online) Time evolution of the expectation value of the witness operator for $T=10 \mathrm{mK}, \alpha=0.007,{ }^{27}$ and $t_{\text {opt }}=20$ (orange dot-dashed line), 10 (green solid line), and $5 \mu \mathrm{eV}$ (black dashed line). Dotted lines mark their asymptotic values. Inset: Energy gap between ground and first excited states in the optimal configuration $\left\{\Delta_{\text {opt }}, t_{\text {opt }}\right\}$ (blue dashed line); the red solid line indicates the thermal energy $k_{B} T=0.86 \mu \mathrm{eV}$.
To study the effect of these charge fluctuations we model the environment as a bosonic bath. ${ }^{28}$ The Hamiltonian of the DQD plus the environment is then given by

$$
\hat{H}=\hat{H}_{D Q D}+\hat{V}_{t} \hat{A}_{t}+\hat{V}_{\Delta} \hat{A}_{\Delta}+\hat{H}_{\mathrm{bath}, t}+\hat{H}_{\mathrm{bath}, \Delta},
$$

where $\quad \hat{V}_{t}=|S(1,1)\rangle\langle S(0,2)|+| S(0,2)\rangle\langle S(1,1)|, \quad \hat{V}_{\Delta}=$ $|S(0,2)\rangle\langle S(0,2)|, \quad \hat{A}_{i}=\sum_{k} a_{i, k}\left(b_{i, k}^{\dagger}+b_{i, k}\right), \quad$ and $\quad \hat{H}_{\mathrm{bath}, i}=$ $\sum_{k} \hbar \omega_{k}\left(b_{i, k}^{\dagger}+b_{i, k}\right)$, with $i=t, \Delta$. The bosonic baths are characterized by symmetric and antisymmetric spectral functions $S^{ \pm}(\omega)$ which are related by $S^{+}(\omega)=\operatorname{coth}\left(\hbar \omega / 2 k_{b} T\right) S^{-}(\omega)$, where $\omega$ is the frequency of the harmonic oscillators of the bath. We assume a bath with Lorentzian damping $S^{-}(\omega)=\alpha \hbar^{2} \omega \frac{1}{1+\left(\omega / \omega_{c}\right)^{2}}$, with $\omega_{c}$ a high cut-off frequency. We also assume weak coupling between the system and the bath and short bath correlation times. Following the Bloch-Redfield approximation, the time-dependent reduced density matrix, written in the basis of the eigenstates of $\hat{H}_{D Q D}$ is given by $\partial_{t} \rho_{a b}=-i \omega_{a b} \rho_{a b}+\sum_{c d} R_{a b c d} \rho_{c d}$, where $\hbar \omega_{a b}=E_{a}-E_{b}$ and $R_{a b c d}$ is the Bloch-Redfield tensor. ${ }^{28}$ The sum in this equation extends over terms with $\omega_{a b}-\omega_{c d} \ll 1 / \Delta \tau$ (the so-called secular constraint), where $\Delta \tau$ is the time scale of the Markovian course-grained evolution. ${ }^{29}$ Considering the initial condition $\rho(0)=\left|\psi_{0}\right\rangle\left\langle\psi_{0}\right|$ the only relevant components of the Bloch-Redfield tensor are $R_{0000}=-R_{1100}$ and $R_{1111}=-R_{0011}$. The time evolutions for the populations of the ground and first excited states are then given by

$$
\begin{gathered}
\rho_{00}(\tau)=\frac{R_{0011}+R_{1100} e^{-\left(R_{0011}+R_{1100}\right) \tau}}{R_{0011}+R_{1100}}, \\
\rho_{11}(\tau)=\frac{R_{1100}\left(1-e^{-\left(R_{0011}+R_{1100}\right) \tau}\right)}{R_{0011}+R_{1100}} .
\end{gathered}
$$

Rewriting the eigenstates given in (3) as $\left|\psi_{i}\right\rangle=$ $\alpha_{i}\left|T_{0}\right\rangle+\beta_{i}\left|S_{11}\right\rangle+\gamma_{i}\left|S_{02}\right\rangle$, the coefficients $R_{i i j j}$ for the optimal values $\left\{\Delta_{\text {opt }}, t_{\text {opt }}\right\}$ are approximately given by $R_{i i j j} \approx 2 G\left(\omega_{j i}\right)\left(\beta_{i} \gamma_{j}+\beta_{j} \gamma_{i}\right)^{2} / \hbar^{2}$ with $G\left(\omega_{i j}\right)=$ $2 \pi^{2} \alpha \hbar^{2} \omega\left[1+\operatorname{coth}\left(\hbar \omega / 2 k_{B} T\right)\right]$. Introducing these expressions in Eqs. (7) and (8), we can obtain the time-dependent probability $P[S(1,1)](\tau)=|\langle S(1,1)|\rho(\tau)| S(1,1)\rangle|$ which, averaged over the nuclear magnetic field probability, provides the expectation value of the entanglement witness plotted in Fig. 2. For these optimal values $P[S(1,1)]$ is approximately given by $P[S(1,1)] \approx \rho_{00}(\tau) \alpha_{1} \beta_{0} \gamma_{2}$.

As can be seen in Fig. 2, the larger the value of the interdot coupling $t$ the longer the system retains a large amount of its initial entanglement. If the ground state is in its optimal configuration $\left\{\Delta_{\text {opt }}, t_{\text {opt }}\right\}$ the loss of entanglement caused by the quantum charge fluctuations in the environment becomes important for time scales of the order of microseconds. These times are much longer than the time scales required to manipulate electrons confined in a DQD [1-100 ns (Ref. 9)], and long enough to adiabatically tune $\Delta$ to its optimum value. This tuning must be done slowly compared to the nuclear mixing time $\sim \hbar / M[\sim 1 \mu$ s (Ref. 11)]. The decay of the entanglement is smaller for $\Delta<\Delta_{\text {opt }}$, allowing for safe adiabatic tuning of $\Delta$. For longer times, the entanglement reaches asymptotic values ranging from $C=0.4(t=5 \mu \mathrm{eV})$ to $C=0.7(t=20 \mu \mathrm{eV})$. Using several DQD's it is possible in 
principle to use distillation of entanglement ${ }^{30}$ to obtain fewer couples of maximally entangled electrons. For these longer times, different sources of decoherence like the spin-orbit interaction $^{32}$ or fluctuations in the nuclear spin configuration ${ }^{23}$ might have a relevant role, and should be explicitly considered.

Conclusion. We have proposed a scheme to detect the entanglement between two electrons in a double quantum dot and shown that it is possible to maximize the entanglement between the two electrons in the ground state of the system. A highly entangled state can be obtained for large tunnel coupling between the dots, with $\Delta$ tuned to its corresponding optimum value. This entanglement can be detected using an entanglement witness, and its expectation value can be easily measured using a quantum point contact. We have also found that both in the optimal configuration that maximizes the entanglement and in its trajectory to this configuration, the entanglement of the ground state is robust against charge fluctuations in the environment.

We would like to thank Fabian Bodoky for useful discussions. This work was supported by the EU project MIDAS.
${ }^{1}$ A. Einstein, B. Podolsky, and N. Rosen, Phys. Rev. 47, 777 (1935).

${ }^{2}$ S. J. Freedman and J. F. Clauser, Phys. Rev. Lett. 28, 938 (1972); A. Aspect, P. Grangier, and G. Roger, ibid. 47, 460 (1981).

${ }^{3}$ M. Lamehi-Rachti and W. Mittig, Phys. Rev. D 14, 2543 (1976).

${ }^{4}$ A. Bramon and M. Nowakowski, Phys. Rev. Lett. 83, 1 (1999).

${ }^{5}$ M. A. Rowe, D. Kielpinski, V. Meyer, C. A. Sackett, W. M. Itano, C. Monroe, and D. J. Wineland, Nature (London) 409, 791 (2001). ${ }^{6}$ Y. Hasegawa, R. Loidl, G. Badurek, M. Baron, and H. Rauch, Nature (London) 425, 45 (2003).

${ }^{7}$ D. L. Moehring, M. J. Madsen, B. B. Blinov, and C. Monroe, Phys. Rev. Lett. 93, 090410 (2004).

${ }^{8}$ M. Steffen, M. Ansmann, R. C. Bialczak, N. Katz, E. Lucero, R. Mcdermott, M. Neeley, E. M. Weig, A. N. Cleland, and J. M. Martinis, Science 313, 1423 (2006).

${ }^{9}$ R. Hanson, L. P. Kouwenhoven, J. R. Petta, S. Tarucha, and L. M. K. Vandersypen, Rev. Mod. Phys. 79, 1217 (2007).

${ }^{10}$ F. H. L. Koppens, C. Buizert, K. J. Tielrooij, I. T. Vink, K. C. Nowack, T. Meunier, L. P. Kouwenhoven, and L. M. K. Vandersypen, Nature (London) 442, 766 (2006); K. C. Nowack, F. H. L. Koppens, Y. V. Nazarov, and L. M. K. Vandersypen, Science 318, 1430 (2007).

${ }^{11}$ J. R. Petta, A. C. Johnson, J. M. Taylor, E. A. Laird, A. Yacoby, M. D. Lukin, C. M. Marcus, M. P. Hanson, and A. C. Gossard, Science 309, 2180 (2005).

${ }^{12}$ R. Hanson and D. Awschalom, Nature (London) 453, 1043 (2008); L. Robledo, H. Bernien, I. van Weperen, and R. Hanson, Phys. Rev. Lett. 105, 177403 (2010).

${ }^{13}$ H. Bluhm, S. Foletti, D. Mahalu, V. Umansky, and A. Yacoby, Phys. Rev. Lett. 105, 216803 (2010).

${ }^{14}$ F. H. L. Koppens, J. A. Folk, J. M. Elzerman, R. Hanson, L. H. Willems van Beveren, I. T. Vink, H. P. Tranitz, W. Wegscheider,
L. P. Kouwenhoven, and L. M. K. Vandersypen, Science 309, 1346 (2005).

${ }^{15}$ W. A. Coish and D. Loss, Phys. Rev. B 72, 125337 (2005).

${ }^{16}$ K. Ono and S. Tarucha, Phys. Rev. Lett. 92, 256803 (2004).

${ }^{17}$ O. N. Jouravlev and Y. V. Nazarov, Phys. Rev. Lett. 96, 176804 (2006).

${ }^{18}$ Y. Tokura, W. G. van der Wiel, T. Obata, and S. Tarucha, Phys. Rev. Lett. 96, 047202 (2006); M. Pioro-Ladrière, T. Obata, Y. Tokura, Y. S. Shin, T. Kubo, K. Yoshida, T. Taniyama, and S. Tarucha, Nature Phys. 4, 776 (2008).

${ }^{19}$ B. M. Terhal, J. Theor. Comput. Sci. 287, 313 (2002).

${ }^{20}$ O. Gühne and G. Tóth, Phys. Rep. 474, 1 (2009).

${ }^{21}$ L. Faoro and F. Taddei, Phys. Rev. B 75, 165327 (2007).

${ }^{22}$ M. Blaauboer and D. P. DiVincenzo, Phys. Rev. Lett. 95, 160402 (2005).

${ }^{23}$ The nuclear spin configuration is considered to be frozen for times of the order of $100 \mu$ s or longer (see Refs. 9 and 31).

${ }^{24}$ We use the convention $i^{1 / 3}=(\sqrt{3}+i) / 2$.

${ }^{25}$ C. H. Bennett, D. P. DiVincenzo, J. A. Smolin, and W. K. Wootters, Phys. Rev. A 54, 3824 (1996).

${ }^{26}$ J. M. Taylor, J. R. Petta, A. C. Johnson, A. Yacoby, C. M. Marcus, and M. D. Lukin, Phys. Rev. B 76, 035315 (2007).

${ }^{27}$ A. Romito and Y. Gefen, Phys. Rev. B 76, 195318 (2007).

${ }^{28}$ U. Weiss, Quantum Dissipative Systems (World Scientific, Singapore, 1999).

${ }^{29}$ C. Cohen-Tannoudji, J. Dupont-Roc, and G. Grynberg, AtomPhoton Interactions (Wiley, New York, 1992).

${ }^{30}$ C. H. Bennett, G. Brassard, S. Popescu, B. Schumacher, J. A. Smolin, and W. K. Wootters, Phys. Rev. Lett. 76, 722 (1996).

${ }^{31}$ L. Chirolli and G. Burkard, Adv. Phys. 57, 225 (2008).

${ }^{32} \mathrm{~A}$ long relaxation time $T_{1} \approx 1 \mathrm{~s}$ caused by the spin-orbit interaction has been measured with $B_{\mathrm{ext}}=1 \mathrm{~T}$ (see Ref. 31 ). 